\title{
Functional results of endoscopic arytenoid abduction lateropexy for bilateral vocal fold palsy
}

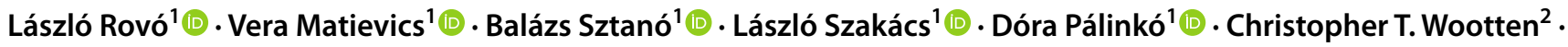

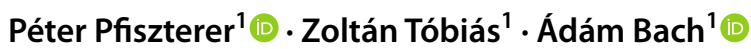

Received: 12 October 2021 / Accepted: 22 November 2021 / Published online: 2 December 2021

(c) The Author(s) 2021

\begin{abstract}
Purpose Endoscopic arytenoid abduction lateropexy (EAAL) is a reliable surgical solution for the minimally invasive treatment of bilateral vocal fold palsy (BVFP), providing a stable airway by the lateralization of the arytenoid cartilages with a simple suture. The nondestructive manner of the intervention theoretically leads to higher regeneration potential, thus better voice quality. The study aimed to investigate the respiratory and phonatory outcomes of this treatment concept.

Methods $61 \mathrm{BVFP}$ patients with significant dyspnea associated with thyroid/parathyroid surgery were treated by unilateral EAAL. Jitter, Shimmer, Harmonics to Noise Ratio, Maximum Phonation Time, Fundamental frequency, Voice Handicap Index, Dysphonia Severity Index, Friedrich's Dysphonia Index, Global-Roughness-Breathiness scale, Quality of Life, and Peak Inspiratory Flow were evaluated 18 months after EAAL.

Results All patients had a stable and adequate airway during the follow-up. Ten patients (16.4\%) experienced complete bilateral motion recovery with objective acoustic parameters in the physiological ranges. Most functional results of the 13 patients $(21.3 \%)$ with unilateral recovery also reached the normal values. Fifteen patients $(24.6 \%)$ had unilateral adduction recovery only, with slightly impaired voice quality. Eleven patients (18.0\%) had false vocal fold phonation with socially acceptable voice. In 12 patients (19.7\%) no significant motion recovery was detected on the glottic level.

Conclusion EAAL does not interfere with the potential regeneration process and meets the most important phoniatric requirements while guaranteeing the reversibility of the procedure - therefore serving patients with transient palsy. Further, a socially acceptable voice quality and an adequate airway are ensured even in cases of permanent bilateral vocal fold paralysis.
\end{abstract}

Keywords Bilateral vocal fold palsy $\cdot$ Endoscopic arytenoid abduction lateropexy $\cdot$ Minimally invasive surgery $\cdot$ Voice quality

\section{Introduction}

Despite the continuous development of surgical techniques, bilateral recurrent laryngeal nerve (RLN) injury mainly occurs as a major complication of thyroid/parathyroid surgery [1]. The position of the vocal folds, general health, cardiopulmonary reserve, and physical activity of a patient mainly determine the severity of dyspnea caused

Ádám Bach

bach.adam@med.u-szeged.hu

1 Department of Otorhinolaryngology-Head and Neck Surgery, Faculty of Medicine, University of Szeged, Tisza Lajos krt. 111, Szeged 6725, Hungary

2 Department of Otolaryngology-Head and Neck Surgery, Vanderbilt University Medical Center, Nashville, TN, USA by consequential bilateral vocal fold palsy (BVFP). Lifethreatening dyspnea obviously requires urgent surgical intervention to prevent acute asphyxiation. Moreover, a patient's quality of life may be adversely affected by mild dyspnea as well; therefore, restoration of adequate airway plays a crucial role in these cases too [2]. Tracheostomy has been accepted for centuries as the gold standard to achieve definitive treatment, despite its potentially severe psychological and somatic adverse effects [3-5]. Continuous improvement of anesthesia and the development of diagnostic and surgical techniques over recent decades have enabled physicians to avoid implementing this highly unpleasant intervention. However, most surgical techniques employ resection of laryngeal structures on some level (e.g., arytenoidectomy and/or cordotomy) having a significant negative impact on voice quality [2,6-8]. Accordingly, these ablative 
procedures are generally applied when permanent paralysis is verified by laryngeal electromyography as well $[9,10]$.

Another surgical approach has arisen since the beginning of the twenty-first century: displacing the vocal fold without tissue resection [7]. These mainly endoscopic procedures achieve vocal fold lateralization using a simple suture. These potentially reversible techniques provide permanent airway restoration in BVFP as well [11, 12]. Although this concept promises simpler management of BVFP, the precise roles of lateralization techniques are still undefined. Further phoniatric studies have yet to evaluate the potential benefits of these techniques, particularly compared with other glottis enlargement procedures.

Endoscopic arytenoid abduction lateropexy (EAAL) is a minimally invasive, quick, and safe intervention for the treatment of vocal fold palsy, that provides an immediate patent airway [13-15]. Via EAAL the arytenoid cartilage can be stabilized in its maximally abducted position in a physiological manner through endoscopically inserted sutures, without resection of delicate phonatory structures. The Endoscopic Thread Guide Instrument (ETGI; Mega Kft, Szeged, Hungary) is designed for accurate, fast and safe suture loop creation for this surgical procedure [13]. The functional results of this surgical technique are reported hereby from the phoniatric and spirometric point of view in cases of temporary and permanent bilateral vocal fold palsy.

\section{Materials and methods}

\section{Patients}

Between January 2017 and December 2018, we sequentially enrolled patients with BVFP associated with thyroid or parathyroid surgery. Unilateral EAAL served as the treatment of choice in all cases to manage their severe/moderate dyspnea. Fifteen patients with thyroid cancer underwent additional radioiodine therapy. Since I-131 therapy does not affect voice quality, the study-group could be considered homogenous from this perspective $[16,17]$. The status of the glottis (particularly the mobility of the vocal folds) was evaluated using a $70^{\circ}$ rigid endoscope during regular control examinations of patients under local anesthesia. The patients were categorized into well-defined groups according to the recovery of vocal fold motion as follows:

- Group I: patients with bilateral complete motion recovery.

- Group II: patients with complete unilateral vocal fold motion recovery.

- Group III: patients with partial vocal fold motion recovery.
- Group IV: patients with false vocal fold phonation without significant active glottic movement.

- Group V: patients without significant motion recovery.

\section{Surgical procedure}

BVFP patients underwent unilateral endoscopic arytenoid abduction lateropexy as described in our earlier publications [13-15]. EAAL was performed under general anesthesia via total intravenous anesthesia and supraglottic jet ventilation. The glottis was explored using a Weerda laryngoscope, and the mobility of the cricoarytenoid joints was bluntly checked to exclude mechanical fixation. The laryngeal mucosa was subsequently disinfected and then the arytenoid cartilage was tilted backward and upward using the tip of the ETGI. The curved, built-in blade was pushed through under the vocal process out to the surface of the neck. During this step, the assistant surgeon led a nonabsorbable suture thread (Prolene 1.0; Ethicon, Somerville, NJ) through the hole at the tip of the blade (Fig. 1a). The blade was then retracted into the laryngeal cavity with the doubled-over thread (Fig. 1b). The arytenoid cartilage was tilted again, and the blade (with the thread) was again extruded above the vocal process to the outer surface of the neck (Fig. 1c). The double-folded thread was cut by the assistant, the blade was retracted into the laryngeal cavity, and the ETGI was finally removed. The threads were transposed under the skin (on the surface of the sternohyoid muscle) into one of the previous incisions, and the corresponding ends were knotted (Fig. 1d).

In cases of appropriate abduction motion recovery (Groups I and II) with sufficient respiratory function, the lateralizing sutures can be removed under local anesthesia. After localization (by palpation) of the suture, an approximately $1-\mathrm{cm}$-long incision is made in the neck. The sutures are cut, the threads are simply removed from the laryngeal lumen, and the incision is closed.

\section{Evaluation of functional results}

Voice assessment was performed 18 months after EAAL, according to our previously published institutional protocol, which was elaborated by the guidelines of the Committee on Phoniatrics of the European Laryngological Society $[18,19]$. The acoustic data were acquired from recordings of three samples of sustained /a:/ at a comfortable pitch and loudness, and a standardized, connected speech sample. Jitter \%, Shimmer \%, Mean Pitch, Harmonics to Noise Ratio (HNR), and Maximum Phonation Times (MPT) were assessed using Praat 6.1.09 software [www.praat.org]. Peak inspiratory flow (PIF) was measured to objectively evaluate respiratory function [20].

As a component of our subjective evaluation, a jury of three physicians and one speech therapist perceptually 
Fig. 1 Left-sided endoscopic arytenoid abduction lateropexy. (schematic drawing, posterior view of the larynx, the arrow shows the movement of the blade). a The built-in, curved blade is pushed through under the vocal process out to the surface of the neck, and a nonabsorbable suture thread is laced through the hole at the tip of the blade. $\mathbf{b}$ The doubled-over thread is pulled back with the blade, into the laryngeal cavity. c After a repeated tilting of the arytenoid cartilage, the blade (and the thread) is pushed out above the vocal process to the outer surface of the neck. d The arytenoid cartilage is stabilized in its maximally abducted position in a physiological manner

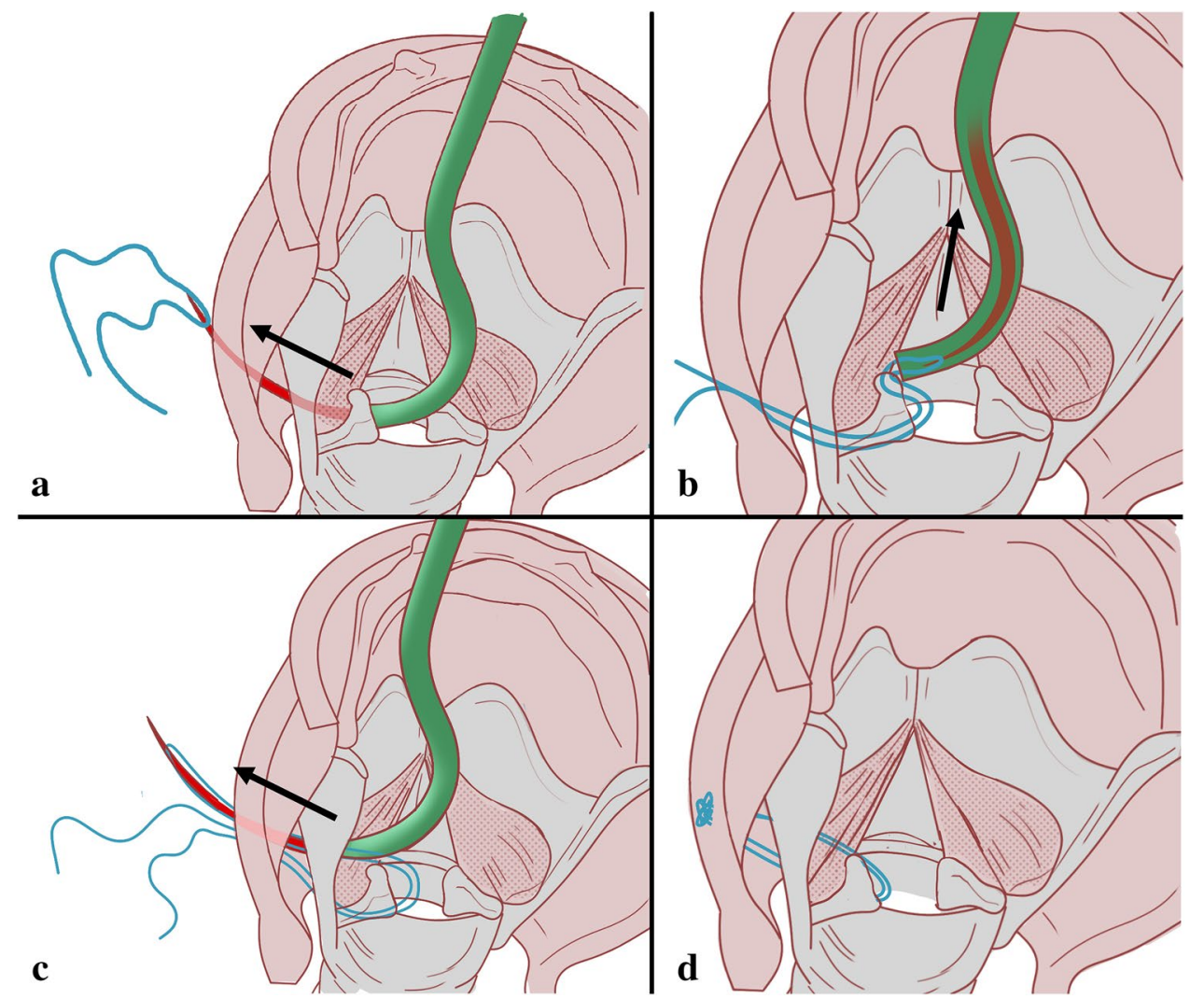

analyzed the connected speech recordings according to the G (global) R (roughness) B (breathiness) criteria (GRBAS) scale [21]. The Dysphonia Severity Index (DSI) [22] and Friedrich's Dysphonia Index (FDI) [23] were also used as complex, calculated linear indexes to evaluate dysphonia. The Hungarian version of the Voice Handicap Index (VHI) was applied for subjective self-evaluation [24]. The functional outcomes of the surgery in terms of breathing, voice, swallowing, and overall satisfaction were assessed using a Quality of Life questionnaire [25].

\section{Results}

We sequentially evaluated 65 patients who were managed for BVFP. Among them, 4 opted out of the study. (Two patients refused the control examinations; two patients died of cardiac arrest.) The remaining 61 patients (49 females [80.3\%] and 12 males [19.7\%]; mean age 52.3 years, range $24-82$ years) underwent phoniatric assessment. Forty-two patients (68.8\%) underwent thyroid surgery 1 day- 6 months before EAAL; 19 patients (31.2\%) underwent the procedure more than 6 months (range 6-36 months) before airway surgery. Five patients were referred to our department intubated, 7 with a tracheostomy and 1 with acute, life-threatening dyspnea. Definitive extubation/decannulation was successful in these cases. In total, all patients had stable and adequate airway during the follow-up as demonstrated in Table 1. Patients did not report swallowing difficulties or aspiration, and none of them required the use of a nasogastric feeding tube. EAAL associated aspiration pneumonia was not diagnosed in the cohort. Accordingly, no sutures were removed for these reasons. Acute laryngitis and/or suture-related inflammation of the neck were registered in 4 patients. (These events occurred in the $3 \mathrm{rd}-6$ th postoperative months.) Repeated EAAL was required for two patients because of spontaneous, partial remedialization of the lateralized vocal fold. Patients' postoperative PIF improvement enabled them to continue their premorbid daily routines and

Table 1 Long-term postoperative spirometric results

\begin{tabular}{lll}
\hline & Preoperative PIF [1/s] & $\begin{array}{l}\text { Late post- } \\
\text { operative } \\
\text { PIF [1/s] }\end{array}$ \\
\hline $\begin{array}{ll}\text { Number of patients } \\
\text { Temporary palsies (Groups }\end{array}$ & $48^{*}$ & 61 \\
I-IV) & $1.65 \pm 0.41$ & $2.83 \pm 0.72$ \\
Permanent palsies (Group V) & & $2.42 \pm 0.40$ \\
\hline
\end{tabular}

Late postoperative measurement $=18$ th month after EAAL

PIF Peak Inspiratory Flow

*13 patients cannot be measured because of tracheostomy, life-threatening dyspnea or intubation 
physical activities. 26 of 61 patients underwent speech therapy sessions after EAAL. The detailed data for voice assessments are presented in Table 2 and are summarized as follows:

- Group I: 10 patients (9 females and 1 male) demonstrated bilateral complete motion recovery (Fig. 2). Among them, the lateralizing sutures were removed during the second through fourteenth (average 5.4 months) postoperative months. The values of relevant objective aerodynamic and acoustic parameters (MPT, HNR, Jitter, Shimmer) were within their physiological ranges, which correlated with the results of perceptual voice analysis and the average score of VHI (12 points). However, DSI (1.42) and FDI (1.08) revealed detectable voice impairment.

- Group II: 13 patients (9 females and 4 males) showed only unilateral complete vocal fold motion recovery. After undergoing EAAL, four patients regained complete activity on the lateralized side, and nine patients' vocal fold regained complete activity on the contralateral side (Fig. 3). The lateralizing sutures of ten patients were removed after 2-6 months (average 3.8 months), although three patients declined removal. Jitter $(0.95 \%)$, Shimmer (3.73\%), and MPT (16.05 s) were within their physiological ranges, and HNR was moderately impaired (13.74 dB). Perceptual analysis and VHI (23.85) demonstrated mild dysphonia. DSI (1.43) and FDI (1.08) showed significant deterioration compared with group $I$.

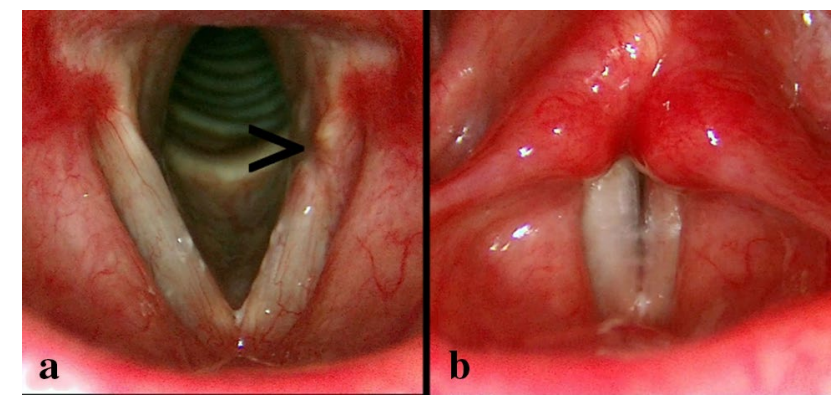

Fig. 2 Endoscopic pictures of a 42-year-old female BVFP patient. In the 8th postoperative month, the left-sided lateralizing sutures were removed due to complete bilateral motion recovery. a Inspiration; $>=$ small mucosal impression at the site of the removed lateralizing suture. b Phonation

- Group III: 15 patients (13 females, 2 males) experienced partial vocal fold motion recovery with predominantly adduction improvement. The value of Shimmer (6.45\%) was particularly higher compared with previous groups. HNR (14.61 dB) and Jitter (1.28\%) were similar to those of group II, despite partial (mainly adduction) regeneration. Perceptual grading, DSI (0.63) and FDI (1.60), indicated deterioration of voice quality, which was not fully reflected by the subjective VHI results (28.40).

- Group IV: 11 patients (8 females and 3 males) experienced false vocal fold phonation without significant active glottic movement. Accordingly, the objective acoustic parameters significantly decreased compared

Table 2 Voice assessment of BVFP patients underwent unilateral EAAL and experienced varying degrees of laryngeal motion recovery

\begin{tabular}{|c|c|c|c|c|c|c|c|c|c|c|c|}
\hline & \multirow{2}{*}{$\begin{array}{l}\text { Aerodynamics } \\
\text { MPT [s] }\end{array}$} & \multicolumn{4}{|l|}{ Acoustics } & \multicolumn{4}{|c|}{ Perception } & \multicolumn{2}{|c|}{ Dysphonia Index } \\
\hline & & Pitch [Hz] & Jitt [\%] & Shim $[\%]$ & $\mathrm{HNR}[\mathrm{dB}]$ & VHI & $\mathrm{G}$ & $\mathrm{R}$ & $\mathrm{B}$ & DSI & FDI \\
\hline Physiological Values & $>15$ & & $<1.04$ & $<3.81$ & $>20$ & $0-120$ & $0-3$ & $0-3$ & $0-3$ & $(-5)-(5)$ & $0-3$ \\
\hline \multicolumn{12}{|c|}{ Group I: patients with bilateral complete vocal fold motion recovery $(n=10 ; 9$ females and 1 male $)$} \\
\hline Mean & 18.09 & 230.31 & 0.73 & 2.49 & 23.42 & 9.0 & 0.50 & 0.3 & 0.2 & 3.43 & 0.60 \\
\hline $\mathrm{SD}( \pm)$ & 4.58 & 86.11 & 0.11 & 0.47 & 3.96 & 3.80 & 0.52 & 0.48 & 0.42 & 1.38 & 0.20 \\
\hline \multicolumn{12}{|c|}{ Group II: patients with unilateral vocal fold motion recovery ( $n=13 ; 9$ females and 4 males) } \\
\hline Mean & 16.05 & 178.09 & 0.95 & 3.73 & 13.74 & 23.85 & 1.23 & 1.23 & 1.15 & 1.42 & 1.08 \\
\hline $\mathrm{SD}( \pm)$ & 5.26 & 75.41 & 0.37 & 0.67 & 4.52 & 8.30 & 1.17 & 1.09 & 0.89 & 0.44 & 0.23 \\
\hline \multicolumn{12}{|c|}{ Group III: patients with partial vocal fold motion recovery ( $n=15 ; 12$ females and 3 males) } \\
\hline Mean & 7.16 & 200.20 & 1.28 & 6.45 & 14.61 & 28.40 & 2.20 & 1.80 & 2.00 & 0.63 & 1.60 \\
\hline $\mathrm{SD}( \pm)$ & 3.62 & 66.11 & 0.21 & 2.87 & 3.48 & 9.87 & 0.86 & 0.94 & 0.85 & 0.98 & 0.27 \\
\hline \multicolumn{12}{|c|}{ Group IV: patients with false vocal fold phonation ( $n=11 ; 8$ females and 3 males) } \\
\hline Mean & 4.32 & 196.19 & 2.15 & 7.02 & 12.62 & 48.18 & 2.64 & 2.00 & 2.64 & -1.48 & 1.82 \\
\hline $\mathrm{SD}( \pm)$ & 1.24 & 55.31 & 0.57 & 4.07 & 2.49 & 12.45 & 0.50 & 0.89 & 0.50 & 0.78 & 0.76 \\
\hline \multicolumn{12}{|c|}{ Group V: patients with no significant motion recovery $(n=12 ; 10$ females and 2 males) } \\
\hline Mean & 3.10 & 177.23 & 3.14 & 12.16 & 9.37 & 72.17 & 2.75 & 2.17 & 2.75 & -2.33 & 2.33 \\
\hline $\mathrm{SD}( \pm)$ & 1.11 & 27.96 & 2.39 & 7.22 & 6.41 & 24.17 & 0.45 & 0.83 & 0.45 & 1.22 & 0.66 \\
\hline
\end{tabular}

MPT maximum phonation time, Jitt Jitter, Shim Shimmer, HNR harmonic to noise ratio, VHI Voice Handicap Index, $G$ global, $R$ roughness, $B$ breathiness (from the GRBAS scale), DSI Dysphonia Severity Index, FDI Friedrich's Dysphonia Index 


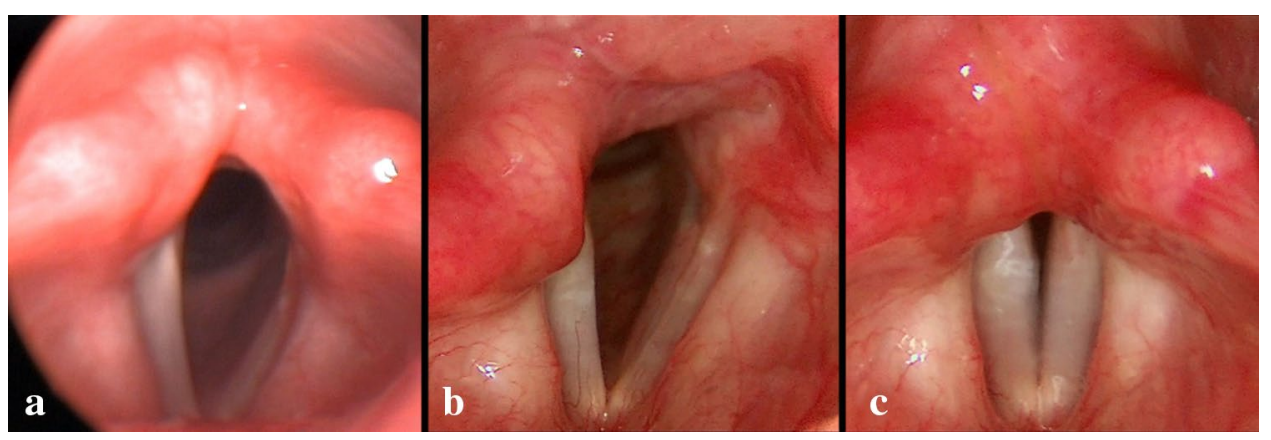

Fig. 3 Endoscopic pictures of a 27-year-old female BVFP patient. a 2 weeks after EAAL on the left side. No significant vocal fold movements were observed. b, c Complete motion recovery of the left (lat-

with those of groups I-III, with an average MPT of $4.32 \mathrm{~s}$. At the same time, subjective parameters, particularly VHI (48.18), revealed poor voice quality.

- Group V: 12 patients (10 females and 2 males) failed to demonstrate significant motion recovery. Thus, these patients were diagnosed with permanent and complete paralysis. Further deterioration was observed, according to objective and subjective parameters (Table 2).

\section{Discussion}

The comparison of the functional outcomes of different surgical techniques addressing BVFP raises a complex question, because the functional results depend on the surgical method as well as a patient's age, sex, mental and physical health, and the potential regeneration of the recurrent laryngeal nerve. Despite numerous studies of humans and animals, insufficient data are available to unambiguously define the pathophysiology of BVFP [26-28]. The intraoperative stretching, mild thermal damage, etc. often cause only axono- or neuropraxy which explains the frequently reported laryngeal function regeneration [1]. However, the exact characterization of functional recovery is rarely cited in the publications connected to the topic of postoperative voice quality $[2,26-30]$.

The mechanisms of neural regeneration are complex and highly variable. For example, physiological reinnervation, pathological/synkinetic reinnervation, and definitive denervation simultaneously occur to varying degrees [28]. These neurological processes may occur several months after the nerve injury, and the definitive functional results may vary from complete, or nearly complete, vocal fold motion recovery to different types of synkinesis, and less frequently to complete neurological immobility. Moreover, nerve anastomoses, which are individual in human larynges or develop eralized) vocal fold. In the 11th postoperative month, the lateralizing sutures were removed. The contralateral fold remained immobile. b Inspiration; c phonation

pathologically during healing, may exert supplementary motor effects on the glottic and supraglottic structures [26, $27,34,35]$. Thus, the requirement for preserving the intrinsic laryngeal muscles during glottis-widening procedures is definitely advantageous even in cases of severe RLN injury because these anatomical structures significantly contribute to residual vocal fold motions and increase the tension of the vocal folds.

The variability of functional recovery indicates that exact categorization of patients according to the result of vocal fold motion recovery is a crucial aspect of the phoniatric evaluation of different glottis-widening techniques. This variability may represent a factor as important as the surgical procedure in determining a patient's postoperative voice. Accordingly, numerous patients with BVFP must be enrolled to create properly powered study groups, which explains the occasionally weak association between voice parameters, even in well-designed surveys [2].

In our present series, 19 of 61 patients (31.1\%), or 33 of 122 vocal folds (27.0\%) showed complete vocal fold motion recovery. Ten patients experienced bilateral recovery with good phonatory closure. These complete motion recoveries were consistently reflected by objective aerodynamic and acoustic parameters. Perceptual voice analysis and calculated indexes were slightly deteriorated, however, thyroidectomies without RLN injuries can have similar impact on voice quality [36]. This anomaly may be explained by the imperfect regeneration of the fine-tuning mechanisms of voluntary vocal fold motions. In total, 19 nonlateralized vocal folds showed complete recovery of motion (complete abduction and adduction). Meanwhile, the same degree of motion recovery was observed in 14 cases on the side of arytenoid lateropexy. This relatively high rate of regeneration of the lateralized vocal folds confirms the noninvasive and consequently reversible aspect of EAAL. It is particularly important to consider that the lateralizing suture was always placed on the side that was assumed to suffer a more severe RLN injury because of previous thyroid/parathyroid surgery. In 
cases of complete motion recovery of the lateralized vocal fold only, good glottic closure was observed after the suture was removed. If motion regeneration occurred on the nonlateralized side, the "released" paretic vocal fold remedialized after the removal of the suture [37]. Accordingly, the objective functional results of patients in Group II nearly reflected physiological values and correlated with the outcomes of patients with unilateral vocal fold palsy who do not undergo glottic surgery $[38,39]$. In our experience, the active and passive mobility of the cricoarytenoid joint remains intact after EAAL. After the removal of the lateralizing suture, no cricoarytenoid joint immobility was observed [40, 41].

Nondynamic parameters of the vocal fold are also critical regarding the voice quality. Such static parameters include the mass, length, and elasticity of the vocal fold [42]. Isshiki et al. (1978) claimed that chaotic vibration patterns and consequential hoarseness can be explained by these unbalanced vocal fold parameters-apart from the inadequate glottic closure. As a minimally invasive procedure, EAAL does not require any resection of the glottic structures. Moreover, the possibility of muscular atrophy is significantly lower because of potential reinnervation. These features of EAAL ensure preservation of the mass of the vocal fold. In addition, EAAL does not damage the membranous part of the vocal fold either. This subregion is principally involved in vibration, and when scarred, voice quality may be compromised. Our previous study of 100 larynges of cadavers found that more tense and straighter vocal folds could be achieved using EAAL compared with other endoscopic glottis-widening procedures [43]. In cases of complete, permanent bilateral immobility, the preserved static components of voice production ensure a sociably acceptable voice with an adequate and stable airway at the same time.

Comparing the late postoperative voice of our patients to their original, 'pre-thyreoidectomy' voice quality would be an instructive study, but preoperative voice assessment is unfortunately not included in the presurgical protocol for thyroid/parathyroid surgery in Hungary. In our experience, we observed notable weakening of voice quality weeks after the onset of the BVFP. We conclude therefore that these early postoperative values cannot provide an exact basis for postoperative phoniatric analysis either. Nevertheless, in contrast to the conventional surgical approaches to BVFP (e.g., transverse cordotomy, partial/total $\mathrm{CO}_{2}$ laser arytenoidectomy, laterofixation via Lichtenberger's needle carrier device), EAAL by ETGI provides better subjective and objective results in patients with BVFP simultaneously with higher peak inspiratory flows (Table 3), [2, 28-32].

Table 3 Long-term postoperative phoniatric parameters of different glottis enlarging procedures

\begin{tabular}{|c|c|c|c|c|c|c|c|c|c|c|c|c|}
\hline & \multirow{2}{*}{$\begin{array}{l}\text { Aerodynamics } \\
\text { MPT [s] }\end{array}$} & \multicolumn{4}{|l|}{ Acoustics } & \multicolumn{4}{|c|}{ Perception } & \multicolumn{3}{|c|}{ Dysphonia Index } \\
\hline & & Pitch $[\mathrm{Hz}]$ & Jitt \% & Shim \% & $\mathrm{HNR}[\mathrm{dB}]$ & VHI & G & $\mathrm{R}$ & $\mathrm{B}$ & DSI & FDI & $\mathrm{PIF}[1 / \mathrm{s}]$ \\
\hline Physiological Values & $>15$ & & $<1.04$ & $<3.81$ & $>20$ & $0-120$ & $0-3$ & $0-3$ & $0-3$ & $(-5)-(5)$ & $0-3$ & \\
\hline \multicolumn{13}{|c|}{ Pruzewicz et al. [29] 13 patients with laser arytenoidectomy (12 females, 1 male) } \\
\hline Mean & & 238.00 & 2.30 & 7.00 & & & & & & & & \\
\hline $\mathrm{SD}( \pm)$ & Not published & & & & & & & & & & & \\
\hline \multicolumn{13}{|c|}{ Dursun et al. [30] 22 patients with transverse cordotomy ( 14 females, 8 males) } \\
\hline Mean & 7.30 & 184.00 & 1.75 & 7.01 & 12.45 & & & & & & & \\
\hline $\mathrm{SD}( \pm)$ & Not published & & & & & & & & & & & \\
\hline \multicolumn{13}{|c|}{ Harnisch et al. [2] 10 patients with mainly transverse cordotomy ( 8 females, 2 males) } \\
\hline Mean & 4.6 & 202.60 & 5.02 & 24.93 & & 55.00 & 2.00 & 1.00 & 2.00 & -5.60 & 2.16 & 1.61 \\
\hline $\mathrm{SD}( \pm)$ & 2.7 & 39.60 & 5.46 & 9.47 & & 19.00 & 1.00 & 1.00 & 1.00 & 6.27 & 0.50 & 0.49 \\
\hline \multicolumn{13}{|c|}{ Yilmaz et al. [31] 20 patients after endoscopic partial/total arytenoidectomy ( 15 females, 5 males) } \\
\hline Mean & $9 / 8$ & $214 / 224$ & & 9.75/9.99 & $8.54 / 8.24 *$ & & & & & & & \\
\hline $\operatorname{SD}( \pm)$ & Not published & & & & & & & & & & & \\
\hline \multicolumn{13}{|c|}{ Lawson et al. [32] 46 patients after subtotal arytenoidectomy/posterior cordectomy ( 28 females, 1 male) } \\
\hline Mean & $6.8 / 7.8$ & & & & & & & & & & & \\
\hline $\operatorname{SD}( \pm)$ & $2.6 / 1.6$ & & & & & & & & & & & \\
\hline \multicolumn{13}{|c|}{$\begin{array}{l}\text { Nawka et al. [33] } 36 \text { patients after permanent transoral surgery (posterior cordotomy, laterofixation, partial arytenoidectomy) ( } 30 \text { females, } 6 \\
\text { males) }\end{array}$} \\
\hline Mean & 7.41 & & 0.84 & & & & 2.0 & 1.5 & 2.0 & -0.11 & & 1.4 \\
\hline $\mathrm{SD}( \pm)$ & 3.67 & & 0.86 & & & & & & & 3.60 & & 0.9 \\
\hline
\end{tabular}

MPT maximum phonation time, Jitt Jitter, Shim Shimmer, HNR Harmonic to Noise Ratio, VHI Voice Handicap Index, $G$ global, $R$ roughness, $B$ breathiness (from the GRBAS scale), DSI Dysphonia Severity Index, FDI Friedrich's Dysphonia Index, PIF Peak Inspiratory Flow

*HNR was calculated as $10 \log (1 / \mathrm{NHR})[45]$ 
In our experience, swallowing problems caused by unilateral EAAL are extremely rare. If present, they are temporary and occur in the early postoperative period only. The minimally invasive EAAL does not damage either the surgically treated or the contralateral vocal fold, and therefore can take advantage of the potential regeneration of the RLN (Groups $I-I I I)$. This way the glottis is surgically opened, but it is able to close during phonation or swallowing processes. After the procedure, the interarytenoid region remains intact as well, which is also essential to safe, aspiration-free swallowing [6]. Furthermore, due to its nondestructive manner, the intervention does not hinder the experience-dependent plasticity of the central nervous system controlling swallowing [44].

\section{Limitations}

This study has certain limitations. Although, the presented objective voice parameters admittedly allow to infer the physical properties of vibration of the vocal fold, laryngeal stroboscopy or high-speed video laryngoscopy may provide further value to the postoperative analysis of the more delicate vocal fold motions. Further, future research should include laryngeal electromyography to stratify the patients according to the severity of their nerve injuries. However, the applied quality of life questionnaire includes a question about swallowing, a specific swallowing-related questionnaire or fiberoptic endoscopic evaluation of swallowing could provide a more detailed evaluation of the functional results.

\section{Conclusion}

The final outcome of BVFP occurs within several months after the onset of recurrent laryngeal nerve injury; thus, BVFP should not be considered a static condition. Accordingly, one of the foundations of the current study was the proper categorization of BVFP patients based on the recovery of vocal fold motions. EAAL - as a quasidynamic surgical solution-provides an immediate adequate airway with reliable long-term results regardless of the recovery of the abduction movements. The procedure which will likely not disturb potential neuroregeneration meets the most important phoniatric requirements because of its minimally invasive manner and guaranteed reversibility. Therefore, EAAL provides beneficial phoniatric outcomes of patients with transient palsy. In cases of bilateral and unilateral motion recovery, the objective voice parameters reached or approximated the physiological ranges. Even patients with exclusively adductive regeneration had only minor voice impairment. Further, EAAL ensured a socially acceptable voice quality and an adequate airway, even for patients with permanent BVFP.
Funding Open access funding provided by University of Szeged. The authors have no funding or financial relationships to disclose.

\section{Declarations}

Conflict of interest The Endolaryngeal Thread Guide Instrument was patented in Hungary in 2008. Patent number: U 07 00163. László Rovó has intellectual property rights pertaining to the device.

Ethics approval We confirm that we have read the Journal's position on issues involved in ethical publication and affirm that this report is consistent with this guideline. Ethical approval for this study was obtained from the Institutional Ethics Committee of the University of Szeged (registration number 162/2019-SZTE)

Consent to participate Informed consent was obtained from all participants of the study.

Consent for publication Each author listed on the manuscript has seen and approved the submission of this version of the manuscript and takes full responsibility for the manuscript.

Open Access This article is licensed under a Creative Commons Attribution 4.0 International License, which permits use, sharing, adaptation, distribution and reproduction in any medium or format, as long as you give appropriate credit to the original author(s) and the source, provide a link to the Creative Commons licence, and indicate if changes were made. The images or other third party material in this article are included in the article's Creative Commons licence, unless indicated otherwise in a credit line to the material. If material is not included in the article's Creative Commons licence and your intended use is not permitted by statutory regulation or exceeds the permitted use, you will need to obtain permission directly from the copyright holder. To view a copy of this licence, visit http://creativecommons.org/licenses/by/4.0/.

\section{References}

1. Rosenthal LH, Benninger MS, Deeb RH (2007) Vocal fold immobility: a longitudinal analysis of etiology over 20 years. Laryngoscope 117:1864-1870

2. Harnisch W, Brosch S, Schmidt M et al (2008) Breathing and voice quality after surgical treatment for bilateral vocal cord paralysis. Arch Otolaryngol Head Neck Surg 134:278-284

3. Halum SL, Ting JY, Plowman EK et al (2012) A multi-institutional analysis of tracheotomy complications. Laryngoscope 122:38-45

4. Goldenberg D, Ari EG, Golz A et al (2000) Tracheotomy complications: a retrospective study of 1130 cases. Review Otolaryngol Head Neck Surg 123:495-500

5. Altinbaş Y, Aslan S, Karaca T (2020) Relationships among body perception, life satisfaction, and attitude toward seeking psychological help in patients with a tracheostomy after discharge: a descriptive and cross-sectional study. Wound Manag Prev 66:32-39

6. Crumley RL (1993) Endoscopic laser medial arytenoidectomy for airway management in bilateral laryngeal paralysis. Ann Otol Rhinol Laryngol 102:81-84

7. Sapundzhiev N, Lichtenberger G, Eckel HE (2008) Surgery of adult bilateral vocal fold paralysis in adduction: history and trends. Eur Arch Otorhinolaryngol 265:1501-1514 
8. Kashima HK (1991) Bilateral vocal fold motion impairment: pathophysiology and management by transverse cordotomy. Ann Otol Rhinol Laryngol 100:717-721

9. Sittel C, Stennert E, Thumfart WF et al (2001) Prognostic value of laryngeal electromyography in vocal fold paralysis. Arch Otolaryngol Head Neck Surg 127:155-160

10. Bach Á, Sztanó B, Kiss JG et al (2018) The role of laryngeal electromyography in the diagnosis of vocal cord movement disorders. Orv Hetil 159:303-311

11. Lichtenberger G (2002) Reversible lateralization of the paralyzed vocal cord without tracheostomy. Ann Otol Rhinol Laryngol 111:21-26

12. Werner JA, Lippert BM (2002) Lateral fixation of the vocal cord instead of tracheotomy in acute bilateral vocal cord paralysis. Dtsch Med Wochenschr 127:917-922

13. Rovó L, Madani S, Sztanó B et al (2010) A new thread guide instrument for endoscopic arytenoid lateropexy. Laryngoscope 120:2002-2007

14. Madani S, Bach Á, Matievics V et al (2017) A new solution for neonatal bilateral vocal cord paralysis: Endoscopic arytenoid abduction lateropexy. Laryngoscope 127:1608-1614

15. Szakács L, Sztanó B, Matievics V et al (2019) Glottic configuration changes and outcomes of endoscopic arytenoid abduction lateropexy. Eur Arch Otorhinolaryngol 276:167-173

16. Isolan-Cury RW, Monte O, Cury AN, Andrada E, Silva MA et al (2008) Acute effects of radioiodine therapy on the voice and larynx of Basedow-Graves patients. Braz J Otorhinolaryngol $74: 224-229$

17. Ribamar do NJJ, Carrara-de Angelis E, Lima ENP (2015) Short term quality of life related to voice and swallowing in patients undergoing ${ }^{131}$ I (iodine) for. Revista CEFAC 17:396-408

18. Dejonckere PH, Bradley P, Clemente P et al (2001) A basic protocol for functional assessment of voice pathology, especially for investigating the efficacy of (phonosurgical) treatments and evaluating new assessment techniques. Guideline elaborated by the Committee on Phoniatrics of the European Laryngological Society (ELS). Eur Arch Otorhinolaryngol 258:77-82

19. Matievics V, Bach Á, Sztanó B et al (2017) Functional outcomes of endoscopic arytenoid abduction lateropexy for unilateral vocal cord paralysis with dyspnea. Eur Arch Otorhinolaryngol 274:3703-3710

20. Cantarella G, Fasano V, Bucchioni E et al (2003) Spirometric and plethysmographic assessment of upper airway obstruction in laryngeal hemiplegia. Ann Otol Rhinol Laryngol 112:1014-1020

21. Wendler J, Rauhut A, Krüger H (1986) Classification of voice qualities. J Phonetics 14:483-488

22. Wuyts FL, De Bodt MS, Molenberghs G et al (2000) The dysphonia severity index: an objective measure of vocal quality based on a multiparameter approach. J Speech Lang Hear Res 43:796-809

23. Friedrich G (1996) Quality assurance in phoniatrics. Recommendation for standardization of clinical voice evaluation] Qualitätssicherung in der Phoniatrie. Vorschlag zur Standardisierung der Klinischen Stimmprüfung. HNO 44:401-416

24. Jacobson BH, Johnson A, Grywalski C et al (1997) The voice handicap index (VHI) development and validation. Am J Speech Lang Pathol 6:66-70

25. Jaquet Y, Lang F, Pilloud R et al (2005) Partial cricotracheal resection for pediatric subglottic stenosis: Long-term outcome in 57 patients. Thorac Cardiovasc Surg 130:726-732

26. Woodson GE (2007) Spontaneous laryngeal reinnervation after recurrent laryngeal or vagus nerve injury. Ann Otol Rhinol Laryngol 116:57-65

27. Crumley RL (2000) Laryngeal synkinesis revisited. Ann Otol Rhinol Laryngol 109:365-371
28. Foerster G, Mueller AH (2021) PCA Atrophy and Synkinesis as the Main Factors for Persistent Vocal Fold Immobility in RLN Paralysis. Laryngoscope 131:E1244-E1248

29. Pruszewicz M, Szmeja Z, Pruszewicz A et al (1995) Voice and spirometric examinations in patients after laser arytenoidectomy. Otolaryngol Pol 49:23-26

30. Dursun G, Gokcan MK (2006) Aerodynamic, acoustic and functional results of posterior transverse laser cordotomy for bilateral abductor vocal fold paralysis. J Laryngol Otol 120:282-288

31. Yilmaz T, Süslü N, Atay G et al (2013) Comparison of voice and swallowing parameters after endoscopic total and partial arytenoidectomy for bilateral abductor vocal fold paralysis: a randomized trial. JAMA Otolaryngol Head Neck Surg 139:712-718

32. Lawson G, Remacle M, Hamoir M et al (1996) Posterior cordectomy and subtotal arytenoidectomy for the treatment of bilateral vocal fold immobility: functional results. J Voice 10:314-319

33. Nawka T, Sittel C, Arens C et al (2015) Voice and respiratory outcomes after permanent transoral surgery of bilateral vocal fold paralysis. Laryngoscope 125:2749-2755

34. Sanders I, Mu L (1998) Anatomy of the human internal superior laryngeal nerve. Anat Rec 252:646-556

35. Wu BL, Sanders I, Mu L et al (1994) The human communicating nerve. An extension of the external superior laryngeal nerve that innervates the vocal cord. Arch Otolaryngol Head Neck Surg 120:1321-1328

36. Van LK, D'haeseleer E, Wuyts FL et al (2010) Impact of thyroidectomy without laryngeal nerve injury on vocal quality characteristics: an objective multiparameter approach. Laryngoscope 120:338-345

37. Rovó L, Jóri J, Brzózka M et al (2000) Airway complication after thyroid surgery: minimally invasive management of bilateral recurrent nerve injury. Laryngoscope 110:140-144

38. SchneiderB DDM, Bigenzahn W (2003) Functional results after external vocal fold medialization thyroplasty with the titanium vocal fold medialization implant. Laryngoscope 113:628-634

39. Siu J, Tam S, Fung K (2016) A comparison of outcomes in interventions for unilateral vocal fold paralysis: a systematic review. Laryngoscope 126:1616-1624

40. Matievics B, Sztanó B, Bach Á et al (2018) Phoniatric outcomes of endoscopic arytenoid abduction lateropexy in patients with transient bilateral vocal cord paralysis. Orv Hetil 159:1188-1192

41. Pálinkó D, Matievics V, Szegesdi I et al (2017) Minimally invasive endoscopic treatment for pediatric combined high grade stenosis as a laryngeal manifestation of epidermolysis bullosa. Int J Pediatr Otorhinolaryngol 92:126-129

42. Isshiki N (2000) Progress in laryngeal framework surgery. Acta Otolaryngol 120:120-127

43. Szakács L, Sztanó B, Matievics V et al (2015) A comparison between transoral glottiswidening techniques for bilateral vocal fold immobility. Laryngoscope 125:2522-2529

44. Ruth EM (2009) Neuroplasticity and swallowing. Dysphagia 24:218-229

45. Godino-Llorente JI, Osma-Ruiz V, Sáenz-Lechón N et al (2008) Acoustic analysis of voice using WPCVox: a comparative study with Multi Dimensional Voice Program. Eur Arch Otorhinolaryngol 265:465-476

Publisher's Note Springer Nature remains neutral with regard to jurisdictional claims in published maps and institutional affiliations. 